\title{
An approximate solution method for boundary layer flow of a power law fluid over a flat plate
}

\author{
T. G. Myers \\ Centre de Recerca de Matemàtica, UAB Science Faculty, \\ 08193 Bellaterra, Barcelona, Spain
}

\begin{abstract}
The work in this paper deals with the development of momentum and thermal boundary layers when a power law fluid flows over a flat plate. At the plate we impose either constant temperature, constant flux or a Newton cooling condition. The problem is analysed using similarity solutions, integral momentum and energy equations and an approximation technique which is a form of the Heat Balance Integral Method. The fluid properties are assumed to be independent of temperature, hence the momentum equation uncouples from the thermal problem. We first derive the similarity equations for the velocity and present exact solutions for the case where the power law index $n=2$. The similarity solutions are used to validate the new approximation method. This new technique is then applied to the thermal boundary layer, where a similarity solution can only be obtained for the case $n=1$.
\end{abstract}

Key words: Boundary layer; power law fluid; heat balance integral method; similarity solutions.

\section{Nomenclature}

$\begin{array}{ll}C & \text { Drag coefficient } \\ H=L / R e^{1 /(n+1)} & \text { Height scale } \\ \mathcal{H} & \text { Convective heat transfer coefficient } \\ L & \text { Plate length } \\ m & \text { Flow consistency index }\end{array}$

Email address: tmyers@crm.cat (T. G. Myers) 


$\begin{array}{ll}n & \text { Power law index } \\ R e & \text { Reynolds number } \operatorname{Re}=\rho U_{\infty}^{2-n} L^{n} / m \\ \operatorname{Pr} & \text { Prandtl number } \operatorname{Pr}=H^{2} U_{\infty} /(\kappa L) \\ Q & \text { Non-dimensional heat flux at } y=0 \\ T & \text { Fluid temperature } \\ T_{\infty} & \text { Far field temperature } \\ \mathbf{u}=(u, v) & \text { Velocity vector } \\ U_{\infty} & \text { Far field velocity } \\ \delta(x), \delta_{T}(x) & \\ \epsilon=\delta_{T} / \delta & \text { Momentum and thermal boundary layer thickness } \\ \kappa & \text { Ratio of boundary layer thicknesses } \\ \rho & \text { Thermal diffusivity } \\ \tau & \text { Fluid density } \\ \xi & \text { Shear stress }\end{array}$

Subscripts

$0 \quad$ Value in fluid at substrate $y=0^{+}$

$s \quad$ Value at substrate $y=0^{-}$

\section{Introduction}

Describing the flow of a Newtonian fluid in the boundary layer above a flat plate is one of the classical problems of fluid mechanics. Since the majority of practical fluids are non-Newtonian the extension of this theory to such fluids is obviously also a key problem. Hence, in this paper the flow of a power law fluid past a flat plate, as well as the associated heat transfer, is examined.

The boundary layer flow of a power law fluid has received much analytical attention, see $[7,8,10,11,12,16]$ for example. When dealing with the momentum boundary layer alone the problem may be analysed using similarity methods. For the Newtonian case the governing equations reduce to the Blasius equation: an ordinary differential equation that is easily solved numerically [26]. For a power law fluid the reduction of the system via similarity variables leads to a modified version of the Blasius equation [11, 12, 25, 27]. 
When the thermal boundary layer is included, due to the differences in the power of the stress gradient and second derivative of temperature, a similarity solution is not possible (except in the Newtonian case). In this case there are two standard ways forward. The governing equations can be solved numerically, see $[2,14,28]$ for example, or via integral methods (which will be discussed in detail later), see $[1,7,8]$. The accuracy of the latter approach is known to deteriorate as the fluid becomes less Newtonian, $[8,12]$. As discussed by Chhabra [8] the numerical results are more accurate than the integral methods but the integral methods are useful since they often lead to closed form solutions. For this reason in the following work we will examine the integral method approach, with a view to improving its accuracy.

In $\S 2$ we derive the governing equations and corresponding integral forms describing the momentum and thermal boundary layers. In $\S 3$ we discuss the similarity solutions for the original and integral forms of the momentum equation. It is shown that both problems have an exact solution for the case where the power law index $n=2$. The numerical solution of the appropriate ordinary differential equations tends to these solutions as $n \rightarrow 2$. In $\S 4$ we describe the standard approximation to the momentum equations attributed to von Kármán and Pohlhausen, see [26], as well as a more accurate method developed by Chhabra $[7,8]$. We then demonstrate a variation of the method designed for the analogous Heat Balance Integral Method that minimises the error introduced by solving the governing equations only in an integral sense, see $[18,21,22,23]$. After demonstrating the improved accuracy of the new method we then apply it to the thermal boundary layer in $\S 5$ for a constant temperature, a constant flux and Newton cooling condition at the plate.

\section{General theory}

The boundary layer equations for two dimensional steady incompressible flow are

$$
\begin{aligned}
u \frac{\partial u}{\partial x}+v \frac{\partial u}{\partial y} & =U_{\infty} \frac{d U_{\infty}}{d x}+\frac{1}{\rho} \frac{\partial \tau}{\partial y} \\
\frac{\partial u}{\partial x}+\frac{\partial v}{\partial y} & =0
\end{aligned}
$$


where $(u, v)$ is the fluid velocity, $U_{\infty}$ is the velocity in the far field and $\tau$ is the shear stress. The velocity profile is subject to the boundary conditions

$$
u=v=0
$$

at $y=0$ and

$$
u=U_{\infty}
$$

as $y \rightarrow \infty$. At $x=0$ the flow is the far field flow, $u(0, y)=U_{\infty}$. For a power law fluid we can set

$$
\tau=m\left|\frac{\partial u}{\partial y}\right|^{n-1} \frac{\partial u}{\partial y}
$$

where $m$ is the consistency index and $n>0$.

If the physical properties of the fluid only depend weakly on the temperature (so that we can assume they are constant) then the momentum boundary layer can be analysed independently of the thermal problem. The thermal problem depends on the flow and for an incompressible fluid is governed by

$$
\frac{\partial}{\partial x}(u T)+\frac{\partial}{\partial y}(v T)=\kappa \frac{\partial^{2} T}{\partial y^{2}}
$$

where $\kappa$ is the thermal diffusivity and $T \rightarrow T_{\infty}$ as $y \rightarrow \infty, T(0, y)=T_{\infty}$. We will discuss the boundary condition at $y=0$ later.

For a Newtonian fluid equations $(1-6)$, with the temperature specified at the plate $T(x, 0)=T_{s}$, can be examined using a similarity variable, see $[9$, p311]. However, when $n \neq 1$ the similarity reduction is not possible so we must resort to numerical or approximate solution methods.

A standard approximation is known as the Integral Momentum Equation (IME), [7]. The IME may be obtained from the boundary layer equations (1, 2 ) or via a simple mass and momentum balance argument, see [7, pp345-351], [26, pp191]. Integrating equation (1) over $y \in[0, h]$, where $h$ is everywhere greater than the boundary layer thickness leads to

$$
\rho \int_{0}^{h} u \frac{\partial u}{\partial x}+v \frac{\partial u}{\partial y}-U_{\infty} \frac{d U_{\infty}}{d x} d y=\left.\tau\right|_{y=0} ^{h} .
$$

We can replace $v$ in the integral via equation (2) after noting $v(x, 0)=0$. This leads to a double integral term; changing the order of integration and integrating once gives

$$
\rho \int_{0}^{h} 2 u \frac{\partial u}{\partial x}-U_{\infty} \frac{\partial u}{\partial x}-U_{\infty} \frac{d U_{\infty}}{d x} d y=-\tau_{0}
$$


where $\tau_{0}$ is the shear stress in the fluid at $y=0$. In the simplest case $U_{\infty}$ is constant and the integral is zero everywhere outside the boundary layer (since then $u=U_{\infty}$ ) and we may replace the upper limit of the integral by the unknown boundary layer thickness $\delta=\delta(x)$ to find

$$
\rho \int_{0}^{\delta} \frac{\partial}{\partial x}\left[u\left(U_{\infty}-u\right)\right] d y=\rho \frac{d}{d x} \int_{0}^{\delta} u\left(U_{\infty}-u\right) d y=\tau_{0} .
$$

This is known as the Integral Momentum Equation (IME). In this form it holds for both laminar and turbulent flow and no assumption has been made about the nature of the fluid [7]. However, from now on we will assume that the power law relation, equation (5), holds. A similar analysis on (6) leads to the Integral Energy Equation (IEE)

$$
\frac{d}{d x} \int_{0}^{\delta_{T}} u\left(T_{\infty}-T\right) d y=\left.\kappa \frac{\partial T}{\partial y}\right|_{y=0}
$$

where the thickness of the thermal boundary layer $\delta_{T}(x) \neq \delta(x)$.

In $\S 4$, when we develop the approximation method, we will work with derivative forms of these equations and so denote $G=\rho u\left(U_{\infty}-u\right), F=$ $u\left(T_{\infty}-T\right)$. Then we will use derivative forms of the integral equations

$$
\begin{array}{ll}
\text { (a) } \frac{\partial G}{\partial x}=-\frac{\partial \tau}{\partial y} \quad \text { (b) } \frac{\partial F}{\partial x}=-\kappa \frac{\partial^{2} T}{\partial y^{2}}
\end{array}
$$

Note, these equations follow from $(9,10)$ by integrating over the boundary layer. For example, with (11b) we note that $F\left(\delta_{T}\right)=T_{y}\left(\delta_{T}\right)=0$ and so

$$
\int_{0}^{\delta_{T}(x)} \frac{\partial F}{\partial x} d y=\frac{d}{d x} \int_{0}^{\delta_{T}(x)} F d y \quad \int_{0}^{\delta_{T}(x)} \frac{\partial^{2} T}{\partial y^{2}} d y=-\left.\frac{\partial T}{\partial y}\right|_{y=0} .
$$

Equation (10) then follows immediately.

\subsection{Non-dimensionalisation}

Using the standard boundary layer scaling for a power law fluid we set

$$
\begin{aligned}
& u=U_{\infty} \hat{u}, \quad v=\frac{U_{\infty}}{R e^{1 /(n+1)}} \hat{v}, \quad x=L \hat{x}, \\
& y=H \hat{y}=\frac{L}{R e^{1 /(n+1)}} \hat{y}, \quad \hat{T}=\frac{T-T_{s}}{T_{\infty}-T_{s}}
\end{aligned}
$$


where $U_{\infty}$ is assumed constant, the Reynolds number $R e=\rho U_{\infty}^{2-n} L^{n} / m$ and $L$ is the plate length. Equation (1) becomes

$$
u \frac{\partial u}{\partial x}+v \frac{\partial u}{\partial y}=\frac{\partial}{\partial y}\left(\left|\frac{\partial u}{\partial y}\right|^{n-1} \frac{\partial u}{\partial y}\right)
$$

where the hats have been dropped. The thermal problem is described by

$$
\operatorname{Pr}\left(\frac{\partial}{\partial x}(u T)+\frac{\partial}{\partial y}(v T)\right)=\frac{\partial^{2} T}{\partial y^{2}}
$$

where the Prandtl number $\operatorname{Pr}=H^{2} U_{\infty} /(\kappa L)$. The IME (9) and IEE (10) become

$$
\begin{aligned}
\int_{0}^{\delta} \frac{\partial}{\partial x}[u(1-u)] d y & =\left(\left[\left|\frac{\partial u}{\partial y}\right|^{n-1} \frac{\partial u}{\partial y}\right]_{y=0}\right)^{n} \\
\operatorname{Pr} \frac{d}{d x} \int_{0}^{\delta_{T}} u(1-T) d y & =\left.\frac{\partial T}{\partial y}\right|_{y=0} .
\end{aligned}
$$

Finally, the derivative forms in (11) are now

$$
\text { (a) } \frac{\partial G}{\partial x}=-\frac{\partial}{\partial y}\left(\left|\frac{\partial u}{\partial y}\right|^{n-1} \frac{\partial u}{\partial y}\right) \quad \text { (b) } \operatorname{Pr} \frac{\partial F}{\partial x}=-\frac{\partial^{2} T}{\partial y^{2}}
$$

where now $G=u(1-u), F=u(1-T)$.

The velocity is subject to the boundary conditions

$$
u(x, 0)=v(x, 0)=0, \quad u(x, \infty)=1, \quad u(0, y)=1 .
$$

The temperature is subject to

$$
T(x, \infty)=T(0, y)=1, \quad \text { (a) } T(x, 0)=0, \text { (b) } T_{y}(x, 0)=Q, \text { (c) } T_{y}=\mathcal{H} T
$$

where $Q$ is a non-dimensional constant heat input and $\mathcal{H}$ is a non-dimensional heat transfer coefficient. We will deal with each of the conditions (21 a, b, c) in $\S 5$. 


\section{Similarity solution for the velocity}

For a Newtonian fluid the similarity solution is obtained by introducing a stream function $(u, v)=\left(\psi_{y},-\psi_{x}\right)$. The stream function may be expressed in terms of the similarity variable $\xi=y / \sqrt{2 x}$, so $\psi=\sqrt{2 x} g(\xi)$ and $g(\xi)$ is an as yet unknown function. Then $u=g_{\xi}$ and $v=\left(\xi g_{\xi}-g\right) / \sqrt{2 x}$. Substituting these into equation (15) leads to the Blasius equation, see, for example, [26, p167-172]. The boundary layer equations for a power law fluid may be derived in a similar manner. The similarity variable is $\xi=y /(n(n+1) x)^{1 /(n+1)}$ and

$$
\begin{aligned}
& u=\psi_{y}=\frac{\psi_{\xi}}{[n(n+1) x]^{1 /(n+1)}}=g_{\xi} \\
& v=-\psi_{x}=\frac{1}{n+1}\left[\frac{n(n+1)}{x^{n}}\right]^{1 /(n+1)}\left(\xi g_{\xi}-g\right) .
\end{aligned}
$$

The modified Blasius equation is then

$$
\frac{\partial}{\partial \xi}\left(\left|\frac{\partial^{2} g}{\partial \xi^{2}}\right|^{n-1} \frac{\partial^{2} g}{\partial \xi^{2}}\right)+n g \frac{\partial^{2} g}{\partial \xi^{2}}=0
$$

which is solved subject to $g(0)=g_{\xi}(0)=0, g_{\xi}(\infty)=1$ (corresponding to $u=v=0$ on $y=0$ and $u \rightarrow 1$ as $y \rightarrow \infty)$. Equation (24) is often written with the modulus sign removed or with all the $g_{\xi \xi}$ terms put together, as written below in equation (25): this requires division by $g_{\xi \xi}^{n-1}$, see $[11,12]$ for example. For $n \leq 1$ it can be shown $g_{\xi \xi}>0[4,16]$ and hence these simplifications are valid. For $n>1, g_{\xi \xi}$ is compactly supported, that is there exists a point $\xi=\xi_{c}>0$ beyond which $g_{\xi \xi}=0$ [4]. If the modulus sign is removed then numerical solutions can predict $g_{\xi \xi}$ becomes negative beyond $\xi_{c}$ and so the velocity will decrease in an unphysical manner. This is in accordance with the observation of $[1,27]$ that the boundary layer has finite thickness. Denier \& Dabrowski [11] show that for $n>1$ a viscous adjustment layer should be introduced to match to the far-field boundary conditions. However, from now on we will neglect the modulus sign in the stress expression and so deal with

$$
\frac{\partial^{3} g}{\partial \xi^{3}}+g\left(\frac{\partial^{2} g}{\partial \xi^{2}}\right)^{2-n}=0 .
$$

This equation holds for all $\xi>0$ when $n \leq 1$ and for $\xi<\xi_{c}$ when $n>1$. Consequently we only deal with physically realistic velocity profiles. 
The similarity solution of the momentum equation (19 a) involves the same variable, $\xi$. Since there is no vertical velocity then there is no need to introduce the stream function and so the governing equation is one order less than (25) (of course since (25) is autonomous we could also reduce the order by one). Setting $u(x, y)=f(\xi)$ and neglecting the modulus sign leads to

$$
\frac{\partial^{2} f}{\partial \xi^{2}}-(1-2 f) \xi\left(\frac{\partial f}{\partial \xi}\right)^{2-n}=0 .
$$

The three conditions $u(x, 0)=0, u(x, \infty)=1, u(0, y)=1$ reduce to $f(0)=0$, $f(\infty)=1$.

The limiting case $n=2$ reduces equation (25) to $g_{\xi \xi \xi}+g=0$. This is a linear constant coefficients equation, with solutions of the form $e^{r x}$, where $r^{3}=-1$. The solution satisfying the boundary condition at $\xi=0$ is

$$
g(\xi)=A_{0}\left[e^{-\xi}+e^{\xi / 2}\left(\sqrt{3} \sin \frac{\sqrt{3} \xi}{2}-\cos \frac{\sqrt{3} \xi}{2}\right)\right] .
$$

Now it is clear that the requirement $g_{\xi}(\infty)=1$ cannot be met and a finite width must be imposed, see [11]. Denoting the edge of the boundary layer as $\xi_{c}$ we set $g_{\xi}\left(\xi_{c}\right)=1$ and introduce a further condition to determine $\xi_{c}$, namely $g_{\xi \xi}\left(\xi_{c}\right)=0$. This leads to

$$
A_{0}=\left[-e^{-\xi_{c}}+e^{\xi_{c} / 2}\left(\sqrt{3} \sin \frac{\sqrt{3} \xi_{c}}{2}+\cos \frac{\sqrt{3} \xi_{c}}{2}\right)\right]^{-1}
$$

where $\xi_{c}$ satisfies the transcendental equation

$$
e^{-\xi_{c}}+2 e^{\xi_{c} / 2} \cos \frac{\sqrt{3} \xi_{c}}{2}=0
$$

In a similar fashion we may solve the Integral Momentum equation (26) for the case $n=2$. Now the solution is in terms of Airy functions with

$$
f(\xi)=a_{0} A i\left(-2^{1 / 3} \xi\right)+a_{1} B i\left(-2^{1 / 3} \xi\right)+\frac{1}{2}
$$

and

$$
\begin{aligned}
& a_{0}=-\frac{3^{2 / 3} \Gamma(2 / 3)}{2}-\sqrt{3} a_{1} \\
& a_{1}=\frac{3^{2 / 3} \Gamma(2 / 3)}{2} \frac{A i^{\prime}\left(-2^{1 / 3} \xi_{c}\right)}{B i^{\prime}\left(-2^{1 / 3} \xi_{c}\right)-\sqrt{3} A i^{\prime}\left(-2^{1 / 3} \xi_{c}\right)}
\end{aligned}
$$


where primes denote differentiation with respect to $\xi$. The position of the edge of the boundary layer $\xi_{c}$ satisfies

$$
a_{0} A i\left(-2^{1 / 3} \xi_{c}\right)+a_{1} \operatorname{Bi}\left(-2^{1 / 3} \xi_{c}\right)=\frac{1}{2} .
$$

\section{Figure 1 near here}

A comparison of the velocities $u(\xi)=f(\xi), g_{\xi}(\xi)$ predicted by the two similarity solutions is shown in Figure 1. The solution to the IME problem, equation (26), is shown as a dashed line whilst that of the original problem, equation (25), is the solid line. The results are shown for four values of $n=0.5,1,1.5,2$. Note, to highlight the differences in the solutions we have truncated the computational domain shown in the figure. For example, when $n=0.5$ we actually carry out the calculation for $g_{\xi}(\xi)$ until $\xi=50$ and for $f(\xi)$ until $\xi=20$. For $n=0.5$ the two solutions are clearly quite different, however, the statement that the IME becomes less accurate as the fluid becomes less Newtonian $[8,12]$ is not strictly true given that the error for $n=$ 1.5 is smaller than that for $n=1$. The lines for $n=2$ show the two analytical solutions, $(27,30)$. Note that, after discussing the fact that the similarity solution does not exhibit the correct far-field behaviour for $n>1$ we still present results for $n=1.5$, where we have taken the end-point as $\xi=5.8$ for the $g$ calculation. In fact up to around $n=1.75$ we are able to produce results where the velocity appears to asymptote to 1 . The solid line for $n=1.5$ shows this quite clearly. Furthermore, the drag coefficient (discussed in $\S 4.5$ ), which is a much stricter indicator of the accuracy, converges to within 3 decimal places. The solutions for $n>1$ exhibit the expected behaviour in that they tend towards the analytical solution for $n=2$ as $n$ increases. So, although the solution with $n>1$ requires a finite-width boundary layer, our calculations indicate that relatively accurate results can be obtained, at least for $n<1.75$, without carrying out any matching to an outer layer.

We now have similarity solutions for the velocity profiles from the full boundary layer equation and the integrated version. When we consider the thermal boundary layer a similarity solution is only possible for the Newtonian case, [9]. This is discussed in $§ 5.1$. Consequently we will now seek approximate solutions that do not have this restriction. The similarity solution will be used to check the accuracy of the approximate solutions. 


\section{Approximate solutions}

\subsection{The Heat Balance Integral Method (HBIM)}

The integral methods described in the following section stem from the seminal work of von Kármán and Pohlhausen [26] on boundary layer flow. However, there has perhaps been more research on the related Heat Balance Integral Method (HBIM) [13, 18]. So, although the HBIM derives from the von Kármán-Pohlhausen method we will briefly describe the HBIM first and then show the analogy with the current problem.

The HBIM is a method for solving the heat equation in an integral sense. If we consider the basic problem of cooling the surface $y=0$ of a semi-infinite material occupying $y>0$ that is initially at a constant temperature. Then we may write the non-dimensional problem as

$$
\frac{\partial T}{\partial t}=\frac{\partial^{2} T}{\partial y^{2}}, \quad T(0, t)=0, \quad T(y, 0)=1, \quad T(\infty, t)=1 .
$$

Although the heat equation has infinite speed of propagation, in the HBIM a distance $\delta$, known as the heat penetration depth, is introduced. For $y \geq \delta$ it is assumed that the temperature rise above the initial temperature is negligible and so $T(\delta, t)=1$ and this replaces the final condition of (34). To ensure that the solutions merge smoothly with the constant outer solution a gradient condition $T_{y}(\delta, t)=0$ is also imposed. Finally, the heat equation is integrated for $y \in[0, \delta]$ to obtain the Heat Balance Integral

$$
\int_{0}^{\delta} \frac{\partial T}{\partial t} d y=\int_{0}^{\delta} \frac{\partial^{2} T}{\partial y^{2}} d y=-\left.\frac{\partial T}{\partial y}\right|_{y=0}
$$

Note that this is rather a weak condition since the choice of $\delta$ only ensures that the area under $T_{t}$ and $T_{y y}$ match. This means that $T_{t}$ can be very different to $T_{y y}$ and so it is no surprise that certain choices of approximating function for $T$ perform significantly better than others [18, 21, 22].

The standard HBIM proceeds by defining a polynomial form of $T$, with coefficients that satisfy the boundary conditions. Substituting the polynomial into (35) then gives a first order ordinary differential equation for $\delta$ which may be solved analytically. A classic issue with this method is the order of the approximating polynomial. Goodman [13] advocates a quadratic. However, cubic and quartic functions have also been used [3, 17, 20, 23]. To improve the accuracy of solutions Braga et al [5] use a non-integer power that 
is chosen based on a known exact solution. To avoid the requirement that approximate solutions should be based on known solutions, thus making the method redundant, Myers [21, 22] developed a method where a non-integer power is chosen based on minimising the error

$$
E=\int_{0}^{\delta}\left[\frac{\partial T}{\partial t}-\frac{\partial^{2} T}{\partial y^{2}}\right]^{2} d y \geq 0
$$

If $T$ is an exact solution then obviously $E=0$. Approximate solutions will have $E>0$. Taking the square of $T_{t}-T_{y y}$ prevents the cancelling of errors of opposite sign and also magnifies the importance of regions where $T$ does not closely satisfy the heat equation [15].

Now consider the current problem. Comparison with the HBIM shows that the IME, equation (17), is an integrated form of solution of equation (19a), where the thickness of the boundary layer is equivalent to the heat penetration depth. Hence if we wish to improve on the accuracy of the IME then we should not only solve the integral equation, equation (17), but also minimize the error

$$
E_{p}=\int_{0}^{\delta}\left(\frac{\partial G}{\partial x}-\frac{\partial \tau}{\partial y}\right)^{2} d y
$$

where $G=u(1-u)$ and the subscript $p$ indicates we take a polynomial power $p$. We will carry this out in $\S 4.3$ but first opt to describe the von Kármán-Pohlhausen Method as a way of introducing the integral method for boundary layer flows.

\subsection{Von Kármán-Pohlhausen Method}

The classical method involves approximating the velocity by a quartic polynomial subject to $u=0$ at $y=0, u=1, u_{y}=u_{y y}=u_{y y y}=0$ at $y=\delta$, hence

$$
u=4 \frac{y}{\delta_{P}}-6\left(\frac{y}{\delta_{P}}\right)^{2}+4\left(\frac{y}{\delta_{P}}\right)^{3}-\left(\frac{y}{\delta_{P}}\right)^{4},
$$

where the subscript $P$ denotes Pohlhausen's solution. Substituting this into the IME, equation (17), without the modulus sign leads to

$$
\frac{d}{d x}\left(\frac{4}{45} \delta_{P}\right)=\left(\frac{4}{\delta_{P}}\right)^{n} .
$$

Applying $\delta_{P}(0)=0$ gives

$$
\delta_{P}=\left(4^{n-1}(n+1) 45 x\right)^{1 /(n+1)} .
$$


Comparison with the Blasius solution shows that Pohlhausen's solution is not a particularly accurate approximation (see $\$ 4.4$ ). Chhabra [8] derives a more accurate representation using a cubic approximating function. The boundary conditions applied by Chhabra are $u=u_{y y}=0$ at $y=0$ and $u=1, u_{y}=0$ at $y=\delta$. The condition $u_{y y}=0$ follows from setting $\mathbf{u}=\mathbf{0}$ in equation (1). Presumably the physical reasoning behind this new boundary condition was based on a desire to improve the accuracy near the wall, $y=0$, with less emphasis on the solution at infinity. Consequently, one boundary condition is dropped at infinity to be replaced by one at $y=0$. This method leads to an approximating function

$$
u=\frac{3}{2} \frac{y}{\delta_{C}}-\frac{1}{2}\left(\frac{y}{\delta_{C}}\right)^{3} .
$$

From the IME we find

$$
\delta_{C}=\left((n+1)\left(\frac{3}{2}\right)^{n} \frac{280}{39} x\right)^{1 /(n+1)}
$$

and this gives significantly more accurate solutions than the previous method (to be discussed further in $\S 4.4$ ).

\subsection{Optimal power solution}

An alternative method is to seek a polynomial approximation where one of the powers is unknown. Taking the same number of terms as in Chhabra's solution we assume an approximating function of the form

$$
u=a_{0}+a_{1}\left(1-\frac{y}{\delta}\right)+a_{2}\left(1-\frac{y}{\delta}\right)^{2}+a_{p}\left(1-\frac{y}{\delta}\right)^{p} .
$$

We expand in terms of $(1-y / \delta)$ for two reasons. Firstly it simplifies the algebra. Secondly, and most importantly, it has been shown for analogous thermal problems that this form provides more accurate solutions than the expansion in $y / \delta[18]$. Applying the same boundary conditions as Chhabra we find

$$
u=1-\frac{p(p-1)}{(p+1)(p-2)}\left(1-\frac{y}{\delta}\right)^{2}+\frac{2}{(p+1)(p-2)}\left(1-\frac{y}{\delta}\right)^{p} .
$$

For large $p$ this reduces to a quadratic form

$$
u \rightarrow\left[1-\left(1-\frac{y}{\delta}\right)^{2}\right]
$$


At the moment we have two unknowns, $p$ and $\delta$. We determine $\delta$ through the IME, equation (17). Evaluating the integral with the velocity of (44) gives

$$
\frac{d}{d x}\left[f_{1}(p) \delta\right]=\frac{f_{2}(p)}{\delta^{n}}
$$

where

$$
\begin{aligned}
& f_{1}(p)=\frac{1}{(p+1)^{2}}\left[\frac{p^{2}+2 p+3}{3}-\frac{2 p^{4}+11 p^{3}+27 p^{2}+25 p+15}{5(p+3)(2 p+1)}\right] \\
& f_{2}(p)=\left(\frac{2 p}{(p+1)}\right)^{n}
\end{aligned}
$$

Hence

$$
\delta=\left[\frac{(n+1) f_{2}}{f_{1}} x\right]^{1 /(n+1)}=\alpha x^{1 /(n+1)},
$$

after applying $\delta(0)=0$. Note, this has the same $x$ dependence as the previous solutions, equations $(40,42)$, only the constant coefficient has changed.

The value of $p$ is determined by minimizing the error $E_{p}$, defined by equation (37), where

$$
\frac{\partial G}{\partial x}=\frac{\partial u}{\partial x}[1-2 u]
$$

The derivatives required to calculate $G_{x}$ and $\tau_{y}$ are

$$
\begin{aligned}
\frac{\partial u}{\partial y} & =\frac{2 p}{(p+1)(p-2) \delta}\left[(p-1)\left(1-\frac{y}{\delta}\right)-\left(1-\frac{y}{\delta}\right)^{p-1}\right] \\
\frac{\partial^{2} u}{\partial y^{2}} & =\frac{2 p(p-1)}{(p+1)(p-2) \delta^{2}}\left[\left(1-\frac{y}{\delta}\right)^{p-2}-1\right] \\
\frac{\partial u}{\partial x} & =\frac{2 p y}{(p+1)(p-2) \delta^{2}} \frac{d \delta}{d x}\left[\left(1-\frac{y}{\delta}\right)^{p-1}-(p-1)\left(1-\frac{y}{\delta}\right)\right] \\
\frac{d \delta}{d x} & =\alpha \frac{x^{-n /(n+1)}}{n+1} .
\end{aligned}
$$

Since the algebra becomes very cumbersome at this stage, we choose to evaluate $E_{p}$ numerically.

\subsection{Velocity results}

The following results were calculated using MATLAB. The error $E_{p}$ was obtained using the derivatives defined in $(51-54)$ and the integral evaluated 
with the subroutine quadl, which uses adaptive Lobatto quadrature. A range of $p$ values was employed and the optimal $p$ chosen to be the one to produce the minimum value of $E_{p}$. The value of $p$ was fixed within $0.1 \%$ of the possible minimum value and $E_{p}$ did not change within 4 decimal places.

\section{Figure 2 near here}

The velocity profiles for a Newtonian fluid are given in Figure 2. The similarity solutions for the boundary layer equations and the IME, equations $(25,26)$, are shown as solid and dashed lines respectively. The optimal $p$ calculation leads to $p=3.48$, which is shown as a dash-dot line and is hard to distinguish from the $p=3$ solution (dotted line). The ' + ' signs show Pohlhausen's solution. These results provide little incentive for using the new method; Chhabra's solution seems sufficiently accurate. However, it is clear that Pohlhausens method and the IME provide the worst approximations. So now we show solutions for $n=0.6$ on Figure 3. Since the velocity profiles in general have the form shown in Figures 1 and 2 we only show a close-up of the solutions in the vicinity of $y=0$. In this case we find the optimal $p=10.016$. Now it is clear that the optimal $p$ solution gives the best approximation to the boundary layer solution. Furthermore, since it provides the best approximation for the velocity near $y=0$ it will also provide the best approximation to the drag coefficient. An interesting point to note is that the IME similarity solution provides the worst approximation and the polynomial solutions are significantly more accurate.

\section{Figure 3 near here}

\subsection{Drag coefficient}

A quantity of primary interest is the drag coefficient $C_{D}$. Since this involves the integral of the shear stress along the plate, any errors in the shear stress will be magnified when calculating $C_{D}$. It is therefore important to have an accurate approximation to the wall stress.

In non-dimensional form the drag coefficient is scaled with $\bar{\tau} /\left(\rho U_{0}^{2}\right)$, where $\bar{\tau}=m U_{0}^{n} / H^{n}$ is the shear stress scale. The drag coefficient is calculated via the shear stress at the substrate

$$
\tau_{0}(x)=\left(\left.\frac{\partial u}{\partial y}\right|_{y=0}\right)^{n}=\left(\frac{g_{\xi}(0)}{[n(n+1) x]^{1 /(n+1)}}\right)^{n} .
$$


The local skin friction coefficient is then defined as $c_{f}(x)=2 \tau_{0}(x)$. The average wall stress is

$$
\bar{\tau}_{0}=\int_{0}^{1} \tau_{0} d x
$$

and the drag coefficient is

$$
C_{D}=2 \bar{\tau}_{0}
$$

So for the similarity solution to the boundary layer equations we find

$$
C_{D}=\frac{2(n+1) g_{\xi \xi}^{n}(0)}{[n(n+1)]^{n /(n+1)}},
$$

where $g_{\xi \xi}(0)$ must be determined numerically. For the similarity solution for the IME we simply replace $g_{\xi \xi}(0)$ with $f_{\xi}(0)$.

For the optimal $p$ solution we use equations (49) and (51) to find

$$
\tau_{0}=\left[\frac{2 p}{(p+1) \alpha}\right]^{n} x^{-n /(n+1)}
$$

where $\alpha=\left((n+1) f_{2} / f_{1}\right)^{1 /(n+1)}$ and $f_{1}, f_{2}$ are defined in equations $(47,48)$. The corresponding drag coefficient is

$$
C_{p}=2(n+1)\left(\frac{2 p}{(p+1) \alpha}\right)^{n} .
$$

Similarly, for Chhabra's solution we can write

$$
C_{C}=2(n+1)\left[\frac{39}{280(n+1)}\left(\frac{3}{2}\right)^{1-n}\right]^{n} .
$$

If we set $p=3$ in (60) then we obtain (61).

In Table 1 we present values for the drag coefficient calculated via the numerical solution of the modified Blasius equation (25) and equation (58) as well as the values calculated through the approximate solutions with $p=3$ and the optimal $p$ method. The values of $C_{D}$ for $n=3,4,5$ are taken from [7]. We do not present results using the IME similarity solution, equation (26), since it is clear from the previous figures that the results are not accurate. Pantokratoras [24] points out that in numerous studies of boundary layer flow the solutions are incorrect due to working over a domain that is too small (in fact he gives over 70 references where the majority have inaccurate 
Table 1: Drag coefficient

\begin{tabular}{cccccc}
\hline$n$ & $C_{D}$ via (58) & $C_{C}$ via $(61)$ & Error & $C_{p}$ via $(60)(p)$ & Error \\
\hline 0.2 & 2.0754 & 1.794 & $13.5 \%$ & 1.868 & $9.9 \%$ \\
0.4 & 1.8377 & 1.626 & $11.5 \%$ & 1.7434 & $5.1 \%$ \\
0.6 & 1.6268 & 1.4914 & $10.7 \%$ & $1.5765(10.016)$ & $4 \%$ \\
0.8 & 1.4597 & 1.3824 & $5.3 \%$ & $1.4239(4.808)$ & $2.4 \%$ \\
1 & 1.3282 & 1.292 & $2.7 \%$ & $1.307(3.488)$ & $1.6 \%$ \\
1.2 & 1.2234 & 1.2183 & $0.4 \%$ & $1.2093(2.74)$ & $1.1 \%$ \\
1.5 & 1.1016 & 1.128 & $-3 \%$ & $1.091(2.114)$ & $0.96 \%$ \\
1.7 & 1.0373 & 1.0781 & $-3.9 \%$ & $1.0282(1.876)$ & $0.9 \%$ \\
2 & 0.959 & 1.014 & $-5.7 \%$ & $0.9513(1.6573)$ & $0.8 \%$ \\
3 & 0.776 & 0.872 & $-12.4 \%$ & $0.786(1.352)$ & $-1.3 \%$ \\
4 & 0.678 & 0.79 & $-16.5 \%$ & $0.6922(1.24)$ & $-2.1 \%$ \\
5 & 0.613 & 0.732 & $-19.4 \%$ & $0.6315(1.18)$ & $-3 \%$ \\
\hline
\end{tabular}

solutions). In particular he points out that a truncated domain will lead to errors in the values of wall shear stress and wall heat transfer. For this reason we have taken great care to ensure that the numerical solution has converged. The numerical solution for $g_{\xi \xi}(0)$ is very sensitive to the length of the domain and so we choose a final value for $\xi$ that is sufficiently large so that the solution does not change within three decimal places. Note, since $\xi=y /[x(n(n+1))]^{1 /(n+1)}$ this position will vary with $n$. For example, if the boundary layer appears to have ended around $y=10$ (and we carry out all calculations at $x=1$ ) then for $n=1$ we compute until $\xi=10 / \sqrt{2}$, whereas for $n=0.4$ we require $\xi \sim 10 /\left((0.2)^{1 / 1.4}\right) \approx 31$ (in fact we take it much further to $\xi=100)$. Acrivos et al [1] obtained values for the drag coefficient that are typically around $5 \%$ different to the present ones. By decreasing our domain we can reproduce their results, indicating that the discrepancy is due to their taking too small a domain for the integration. In the first column of Table 1 we show the $n$ value, then in the second column the drag coefficient, $C_{D}$, calculated from the similarity solution, (58). The third and fourth columns give the drag coefficient calculated using Chhabra's solution with $p=3$ and the associated difference with the similarity solution. The 
fifth and sixth columns show the drag coefficient using the optimal $p$ method and the error. In brackets is the $p$ value required to obtain this solution. Using the optimal $p$ method we cannot obtain solutions for $n<0.555$. The problem appears to be that as $n$ decreases $p$ increases and the velocity profile becomes approximately quadratic, with a very slight deviation near $x=0$. Consequently the higher order polynomial is not appropriate. So, for small $n$ the velocity may be described by the quadratic form $(45)$ where $\delta(x)$ given by (49) takes the limiting value $\alpha \rightarrow\left(15(n+1) 2^{n-1}\right)^{1 /(n+1)}$. This leads to $C_{p} \rightarrow 2(n+1)(2 / \alpha)^{n}$ as $p \rightarrow \infty$. Unfortunately, since the deviation from quadratic is in the vicinity of $y=0$ the greatest errors are also there and so the drag coefficient calculation deteriorates, although they are still an improvement on choosing $p=3$.

From the table it can be seen that in general the current method provides a significantly more accurate estimate of the drag coefficient than when using $p=3$, with the exception of a small region where the error goes from positive to negative (and consequently must somewhere be zero). When a $p$ value can be found the error is always below $4 \%$. This increases to a maximum of around $10 \%$ for the lowest value of $n$, when the infinite $p$ approximation is used. Taking $p=3$ the maximum error is around $20 \%$ and half the results shown have an error over $10 \%$. The differences in the models can be seen more clearly in Figure 4. This shows the drag coefficient calculated by the similarity solution (solid line), equation (58), the optimal $p$ solution (dashed line) and that of equation (61) (*'s). The kink in the dashed line at $n \approx 0.5$ indicates the transition to the limiting formula. For $n>0.5$ the current method is clearly very accurate, whilst that with $p=3$ shows a solution which diverges from the numerical one. The assertion that the approximate solution loses accuracy as the fluid becomes less Newtonian $[8,12]$ does not hold for the current method; the solution accuracy is really only lost for $n<0.5$.

\section{Figure 4 near here}

From Table 1 it is clear that the optimal $p=p(n)$. Obviously it is undesirable (impractical) to calculate $p$ for every case. Noting that $p$ increases rapidly as $n \rightarrow 0$ we look for an approximation of the form $p_{a}=\sum a_{i} / n^{i}$. Defrawi \& Finlayson [10] propose a similar approximation $p \sim 1+1 / n$. Since no real fluid has $n>2$ we use fifteen data points for $n \in[0.57,2]$ to obtain the curve fit (seven of these points are given in Table 1). Using these data 
points a generalised linear approximation $p_{a}=a_{0}+a_{1} / n$ gives an error

$$
E=\sum_{n=1}^{5}\left(\frac{p-p_{a}}{p}\right)^{2} \approx 1.77
$$

Whereas if we use an approximation up to $n^{-4}$ we find an error of 0.05 . We therefore take

$$
p_{a}=22.13-\frac{98.3}{n}+\frac{165.6}{n^{2}}-\frac{114.8}{n^{3}}+\frac{29.01}{n^{4}} .
$$

Using this to calculate $p$ for $n \leq 0.5$ gives almost exact agreement with the $C_{p}$ obtained by letting $p \rightarrow \infty$. The highest error occurs for $n=0.6$ where we find $p=10.74$ and the error in $C_{p}$ is then $2.8 \%$ (which is in fact an improvement on the result from the exact calculation).

\section{Thermal boundary layer}

The thermal problem is governed by equation (16) or the IEE, equation (18). Since the right hand side involves $T_{y y}$, whereas the momentum equation has $\tau_{y}^{n}$, a similarity solution for the coupled problem can only be found for the case $n=1$ and with a fixed temperature at $y=0$. At this stage the approximate solution methods are invaluable. Having verified in the previous section that the optimal $p$ method provides the most accurate results, we will only use this method to determine the temperature profile.

We begin by analysing the case where the temperature is fixed at the substrate and then briefly describe the extensions to constant flux and Newton cooling conditions.

\subsection{Similarity solution for the temperature}

The similarity variable of $\S 3$ may be applied to the temperature equation (16). To balance the right hand side (the $T_{y y}$ expression) with the left hand side (involving the velocity expressions) requires $n=1$. In which case the governing heat equation becomes

$$
\frac{\partial^{2} T}{\partial \xi^{2}}=-\operatorname{Prg} \frac{\partial T}{\partial \xi}
$$


where $\xi=y / \sqrt{2 x}$ and $u=g_{\xi}$ is the solution of (25) with $n=1$. This is to be solved subject to $T(0)=0, T(\infty)=1$. For the Integral Momentum equation, the equivalent expression is

$$
\frac{\partial^{2} T}{\partial \xi^{2}}=\operatorname{Pr} \xi\left[\frac{\partial f}{\partial \xi}(1-T)-f \frac{\partial T}{\partial \xi}\right] .
$$

where $u=f(\xi)$ is the solution of equation (26) with $n=1$.

\subsection{Fixed temperature substrate}

First consider the boundary conditions $T(0, y)=T(x, \infty)=1, T(x, 0)=0$. For the integral method the conditions on $y$ translate to $T\left(x, \delta_{T}\right)=1$, $T_{y}\left(x, \delta_{T}\right)=0, T(x, 0)=0, \delta_{T}(0)=0$. Chhabra uses a physical argument to derive a further boundary condition $T_{y y}=0$. We may derive this in a less intuitive way from the governing equation, namely (16), by writing it as

$$
\operatorname{Pr}(T \nabla \cdot \mathbf{u}+\mathbf{u} \cdot \nabla T))=\frac{\partial^{2} T}{\partial y^{2}} .
$$

For an incompressible fluid $\nabla \cdot \mathbf{u}=0$ and on the boundary $y=0$ the velocity is $\mathbf{u}=\mathbf{0}$. Consequently $T_{y y}=0$ and this is independent of the boundary condition on $T$ at $y=0$.

With the extra boundary condition we may specify a temperature similar to the velocity profile of equation (44),

$$
T=1-\frac{q(q-1)}{2} a_{q}\left(1-\frac{y}{\delta_{T}}\right)^{2}+a_{q}\left(1-\frac{y}{\delta_{T}}\right)^{q},
$$

where $a_{q}=2 /((q+1)(q-2))$.

When calculating the IEE the algebra becomes very unwieldy. So, we follow Chhabra [7] and note that the thermal boundary layer is thinner than the momentum boundary layer, hence we set $\delta_{T} / \delta=\epsilon \ll 1$ (we will discuss this later). The integral on the left hand side of (18) may be written as

$$
\int_{0}^{\delta_{T}} u(1-T) d y=\frac{(q-2)(q+3)\left(q^{2}+q+4\right)}{12(2+q)(1+q)} \frac{p}{(1+p)} a_{q} \delta_{T} \epsilon+\mathcal{O}\left(\epsilon^{2}\right),
$$

where it should be noted that the leading order terms in $\epsilon$ have cancelled out. Whilst the right hand side of (18) becomes

$$
\left.\frac{\partial T}{\partial y}\right|_{y=0}=\frac{q(q-2) a_{q}}{\delta_{T}} .
$$


Hence the IEE may be written as

$$
\operatorname{Pr} \frac{d}{d x}\left(\lambda_{1} \epsilon^{2} \delta\right)=\frac{2 q}{1+q} \frac{1}{\epsilon \delta}
$$

where

$$
\lambda_{1}=\frac{(q+3)\left(q^{2}+q+4\right)}{6(2+q)(1+q)^{2}} \frac{p}{(1+p)} .
$$

The momentum boundary layer thickness $\delta$ is given by equation (49), $\delta=$ $\alpha x^{1 /(n+1)}$, and so we may write equation (70) in the form

$$
\mu_{1} \epsilon x^{1 /(n+1)} \frac{d}{d x}\left(\epsilon^{2} x^{1 /(n+1)}\right)=1
$$

where $\mu_{1}=\operatorname{Pr} \lambda_{1} \alpha^{2}(1+q) /(2 q)$. This may be solved analytically to determine $\epsilon(x)$. However, since $\delta_{T}(0)=\delta(0)=0$ we find $\epsilon(0)$ is undefined. To avoid this issue we set $\delta_{T}\left(x_{0}\right)=0$ and then let $x_{0} \rightarrow 0$ to obtain

$$
\delta_{T}=\alpha\left(\frac{3(n+1)}{\mu_{1}(2 n+1)}\right)^{1 / 3} x^{(n+2) /(3(n+1))} .
$$

Finally, the problem has reduced to determining the single unknown $q$. This is achieved by minimizing the error

$$
E_{q}=\int_{0}^{\delta_{T}}\left(\frac{\partial F}{\partial x}-\frac{\partial^{2} T}{\partial y^{2}}\right)^{2} d y
$$

where $F=u(1-T)$.

This calculation turns out to be much simpler than in the momentum boundary layer solution. The value of $q$ for $n>0.5$ is relatively constant (around 13), for $n<0.5$ the value increases significantly, for example, for $n=0.3, q \approx 35$. Consequently we may obtain accurate solutions by assuming $q \gg 1$ and so the temperature is approximately quadratic

$$
T \approx \frac{y}{\delta_{T}}\left(2-\frac{y}{\delta_{T}}\right)
$$

Note, if we neglect the extra boundary condition $T_{y y}(0, t)=0$ and impose a temperature profile $T=1-\left(1-y / \delta_{T}\right)^{q}$ then it turns out that $q \approx 2$ and so the profiles are equivalent. 
In the limit of large $q$ the constants required to calculate $\delta_{T}$ are

$$
\lambda_{1} \approx \frac{p}{6(p+1)}, \quad \quad \mu_{1} \approx \frac{\operatorname{Pr} p \alpha^{2}}{12(p+1)}
$$

and $\alpha$ is defined in equation (49).

In Figure 5 we compare the temperature profiles at $x=1$, when $n=1$, predicted by the similarity solution, equation (64), the IEE similarity solution, equation (65), and the optimal $q$ solution, equation (67), with $q \approx 13$. For $T<0.6$ the optimal $q$ solution clearly gives excellent agreement with the solution of equation (64). For larger $T$ it slightly overestimates the temperature. The IEE solution overestimates the temperature for small $T$ and underestimates it for larger $T$. Note, this case where $n=1$ is the only one where we can find a similarity solution to the full problem. For all other values we must rely on the approximate solutions.

\section{Figure 5 near here}

In Figure 6 we present the temperature profiles at $x=1$ calculated by optimizing $q$ for the profile (67) and by using the simpler quadratic approximation to the temperature, equation (75). The parameter values are similar to those used in the example in [7, pp363-364], $m=0.3, \kappa=1.4 \times 10^{-7}, \rho=$ $10^{3}, L=0.5, U_{\infty}=2$ (in SI units) but with a range of $n=0.5,1,1.5$. The height-scale $H$ depends on $n, H=L / R e^{1 /(n+1)}$ and consequently so does the Prandtl number $\operatorname{Pr}=H^{2} U_{\infty} /(\kappa L)$. For the three $n$ values we find $(H, P r)=(0.0014,56.74),(0.0087,2136),\left(0.026,1.9 \times 10^{4}\right)$. The value of $p$ calculated from the momentum problem is $p=\infty, 3.49,2.114$ which determines $\alpha=6.33,4.76,3.74$. From the figure we can see that the agreement is good between all sets of curves, giving confidence in the quadratic approximation. Consequently, to determine the temperature profile in the thermal boundary layer it is sufficient to specify $T$ as a quadratic, through equation (75), with $\delta_{T}$ calculated via (73) and $\mu_{1}$ from (76). To be specific, for the thermal problem there is no need to search for an optimum $q$.

\section{Figure 6 near here}

In carrying out the preceding analysis, following [7], we made the assumption that $\delta_{T} \ll \delta$. The variation of these two functions for $n=0.5,1,1.5$ is shown in Figure 7 , where $\delta_{T}$ is the dashed line and $\delta$ the solid line. Taking the 
definitions of $\delta, \delta_{T}$ we find that the assumption reduces to the requirement that

$$
x^{1-n} \gg\left(\frac{3(n+1)}{\mu_{1}(2 n+1)}\right)^{n+1} .
$$

So, for $n<1$ there will always be a region close to $x=0$ where this assumption is violated. However, when we calculate this value we find that $n=0.5$ leads to $x \gg 2 \times 10^{-6}$ and this value decreases as $n$ increases. So, although the assumption is invalid near $x=0$, the region where it is violated is negligible. In fact, many of the model assumptions are suspect in the vicinity of $x=0$, so this current problem should not be viewed too negatively. For example, the plate is treated as infinitely thin: for a finite thickness plate the velocity profile will be rather different where the fluid first contacts the plate. For $x<0$ the velocity gradient is zero and so, for $n<1$ the power law model predicts infinite viscosity, whereas for $n>1$ the viscosity is zero [19]. Once the plate is reached there is, apparently, an immediate change to finite values. In the following examples with different conditions on the temperature at $y=0$ we will not show the development of the momentum and thermal boundary layers because they are all approximately the same as those shown in Figure 7.

\section{Figure 7 near here}

\subsection{Constant flux and Newton cooling boundary conditions}

For completeness we now summarize the analysis for constant flux and Newton cooling boundary conditions.

For constant flux the problem is now subject to $T_{y}(x, 0)=Q, T\left(x, \delta_{T}\right)=$ $1, T_{y}\left(x, \delta_{T}\right)=0, T_{y y}(x, 0)=0$. The analysis follows that in $\S 5.2$. The temperature is defined by equation (67) but now $a_{q}=\delta_{T} Q /(q(q-2))$. The integral (68) and derivative (69) remain the same and the IEE becomes

$$
\mu_{2} \frac{d}{d x}\left(\epsilon^{3} x^{2 /(n+1)}\right)=1
$$

where

$$
\mu_{2}=\operatorname{Pr}\left[\frac{(q+3)\left(q^{2}+q+4\right)}{12 q(2+q)(1+q)} \frac{p}{(1+p)}\right] \alpha^{2}
$$

This gives

$$
\delta_{T}=\alpha \mu_{2}^{-1 / 3} x^{(n+2) /(3(n+1))} .
$$


Evaluating $E_{q}$, again we find that $q \gg 1$ and so the problem simplifies considerably. The temperature is then given by

$$
T \approx 1-\frac{\delta_{T} Q}{2}\left(1-\frac{y}{\delta_{T}}\right)^{2}
$$

with $\delta_{T}$ specified by (80) and $\mu_{2} \rightarrow \mu_{1}$ which is defined by equation (76b).

\section{Figure 8 near here}

In Figure 8 we compare the temperatures for the constant flux problem using the optimal $q$ and the quadratic approximation. In this figure the difference can only be seen for a very small region close to $y=0$ for the case $n=0.5$, otherwise the curves are indistinguishable. This is consistent with the results of [21], where it was shown that for a standard thermal problem the constant flux approximation was more accurate than for a fixed temperature.

A Newton cooling condition at the substrate leads to $T_{y}(x, 0)=\mathcal{H} T(x, 0)$. With the remaining boundary conditions the temperature is given by (67) where $a_{q}=2 \mathcal{H} \delta_{T} /\left((q-2)\left(2 q+\mathcal{H} \delta_{T}(q+1)\right)\right)$. The IEE follows from the same results as given by equations $(68,69)$ but with the new expression for $a_{q}$. This case shows a key difference to the previous two cases in that the IEE becomes

$$
\operatorname{Pr} \frac{d}{d x}\left(\lambda_{2}(p, q, \delta) \epsilon^{3} \delta^{2}\right)=\frac{q}{2 q+\epsilon \delta \mathcal{H}(1+q)}
$$

where

$$
\lambda_{2}=\frac{(q+3)\left(q^{2}+q+4\right)}{12(2+q)(1+q)(2 q+\epsilon \delta \mathcal{H}(1+q))} \frac{p}{(1+p)}
$$

The function $\lambda_{2}$ depends on $\delta$ and the IEE cannot be solved analytically. However, in the case where $q \gg 1$ and $\epsilon \delta \mathcal{H}=\delta_{T} \mathcal{H} \ll 2$ then

$$
\lambda_{2} \rightarrow \lambda_{2}^{\prime}=\frac{(q+3)\left(q^{2}+q+4\right)}{24 q(2+q)(1+q)} \frac{p}{(1+p)}
$$

and the problem reduces to solving

$$
\mu_{3} \frac{d}{d x}\left(\epsilon^{3} x^{2 /(n+1)}\right)=1
$$


where $\mu_{3}=2 \operatorname{Pr} \lambda_{2}^{\prime} \alpha^{2}$. This leads to

$$
\delta_{T}=\alpha \mu_{3}^{-1 / 3} x^{(n+2) /(3(n+1))} .
$$

When we minimise $E_{q}$ the error decreases monotonically as $q$ increases, with an asymptote of around 0.063 . In previous examples $E_{q}$ had a distinct minimum. Consequently we may assume that once again $q$ is large and so take a quadratic approximation to the temperature

$$
T \approx 1-\frac{\mathcal{H} \delta_{T}}{2}\left(1-\frac{y}{\delta_{T}}\right)^{2} .
$$

Note that the temperature stays positive since we have assumed that $\mathcal{H} \delta_{T} \ll 2$. As with the constant flux condition the constant in the expression for $\delta_{T}, \mu_{3} \rightarrow \mu_{1}$ (defined by equation (76b)) and consequently the non-dimensional thermal boundary layer thickness is the same for all three thermal boundary conditions at $y=0$. Figure 7 therefore serves to describe the boundary layer growth for all three thermal boundary conditions.

In Figure 9 we show the temperature for the Newton cooling condition with $n=0.5,1,1.5$. Only the quadratic approximation is shown since as mentioned above $E_{q}$ decreases with $q$ and hence $q \rightarrow \infty$. The dimensional heat transfer coefficient is taken as $10 \mathrm{~W} / \mathrm{m}^{2} \mathrm{~K}$ which leads to $\mathcal{H}=0.024,0.15$, 0.43. As can be seen from Figure 7 the maximum value of $\delta_{T}$ is around 0.2 for $n=1.5$ and 1.2 for $n=0.5$ hence $\delta_{T} \mathcal{H} \ll 2$ is easily satisfied. As with all other examples the temperature for the shear thinning fluid grows most rapidly.

\section{Figure 9 near here}

\section{Conclusion}

In this paper we have considered a number of aspects of the boundary layer flow of a power law fluid. Firstly, we considered the similarity solutions for the velocity profile using the standard boundary layer equations and the Integral Momentum equation. For both cases an exact solution was presented for the case $n=2$. Of particular interest is the fact that although strictly speaking the similarity solutions for $n>1$ should be patched onto an intermediate region, the results for $n<1.75$ appear to be accurate without this extra region. 
For a Newtonian fluid and a constant temperature boundary condition similarity solutions can be found for the coupled velocity and temperature equations. For other values of $n$ and temperature boundary conditions we used the polynomial approximations. For the velocity profile we found $u \sim$ $(1-y / \delta)^{p}$, where $p$ turned out to depend on $n$. The value of $p$ may be calculated by minimising the error or by using a relation of the form $p=$ $\sum_{n=0}^{4} a_{i} / n^{i}$. In all cases examined the temperature is approximately quadratic.

It was shown that the Heat Balance Integral Method was analogous to the Integral Momentum equation. Hence the recently developed method for minimising the error in the HBIM could be applied to this problem. The new method does not suffer from problems associated with $n>1$, namely that the velocity gradient can become negative, and hence results were obtained for $n$ up to 5 (we stopped there only because this was the highest value we could find in the literature). From the results we saw that the standard IME generally provides rather inaccurate results. Using the new integral method reduces the error considerably and so, hopefully, makes this a viable alternative to the full numerical solution.

\section{Acknowledgements}

I would like to thank Prof. Chhabra of IIT Kanpur for his advice and very importantly for sending me many appropriate reference works on this subject.

\section{References}

[1] A. Acrivos, M.J. Shah, E.E. Petersen, Momentum and heat transfer in laminar boundary flow of non-Newtonian fluids past external surfaces, AIChE J. 6 (1960) 312 - 317.

[2] H.I. Andersson and T.H. Toften, Numerical-solution of the laminar boundary-layer equations for power-law fluids, J. Non-Newt. Fl. Mech. 32(2) (1989), 175-195.

[3] A. Antic and J.M. Hill, The double-diffusivity heat transfer model for grain stores incorporating microwave heating, Appl. Math. Modelling, 27 (2003), 629-647. 
[4] M. Benlahsen and M. Gueddab and R. Kersner, The Generalized Blasius equation revisited, Math. and Comp Modelling, 47(9-10) (2008), 10631076.

[5] W. F. Braga, M. B. H. Mantelli, and J. L. F. Azevedo, Approximate analytical solution for one-dimensional finite ablation problem with constant time heat flux, AIAA Thermophys. Conference, 2004.

[6] T. Cebeci and P. Bradshaw, Momentum transfer in boundary layers, McGraw-Hill 1977.

[7] R.P. Chhabra and J.F. Richardson, Non-Newtonian flow and applied rheology, 2nd edition, Butterworth-Heineman 2008.

[8] R.P. Chhabra, Laminar boundary layer heat transfer to power law fluids: an approximate analytical solution, J. Chem. Engng Japan 32(6) (1999), 812-816.

[9] N.P. Cheremenisoff (Ed.), Encyclopedia of Fluid Mechanics, Vol. 7 Rheology and non-Newtonian flows, Gulf Publishing 1988.

[10] M. El Defrawi and B. A. Finlayson, On the use of the integral method for flow of power law fluids, AIChE J. 18 (1972), 251-253.

[11] J. P. Denier and P. P. Dabrowski, On the Boundary-Layer Equations for Power-Law Fluids, Proc. R. Soc. Lond. A460 (2004), 3143-3158.

[12] C-C Hsu, A simple solution for boundary layer flow of power law fluids past a semi-infinite flat plate. AIChE J. 15(3) (1969), 367-370.

[13] T.R. Goodman, The Heat-Balance Integral and its application to problems involving a change of phase, Trans. ASME 80 (1958), 335-342.

[14] M.J. Huang and C.K. Chen, Numerical-analysis for forced-convection over a flat-plate in power law fluids, Int. Comm. Heat Mass Trans. 11(4) (1984), 361-368.

[15] D. Langford, The Heat Balance Integral Method, Int. J. Heat \& Mass Trans., 16 (1973), 2424-2428. 
[16] P.F. Lemieux, R.N. Dubey and T.E. Unny, Variational method for a pseudoplastic fluid in a laminar boundary layer over a flat plate, Trans. ASME J. Appl. Mech. June (1971) 345-349.

[17] S. L. Mitchell and T. G. Myers, Heat balance integral method for onedimensional finite ablation, AIAA J. Thermophys. \& Heat Trans., 22(3) (2008), 508-514.

[18] S.L. Mitchell and T.G. Myers, The application of standard and refined heat balance integral methods to one-dimensional Stefan problems, to appear SIAM Review.

[19] T.G. Myers, The application of non-Newtonian models to thin film flow, Physical Rev. E, 72 (2005) 066302-1-11, DOI: 10.1103/PhysRevE.72.066302.

[20] T.G.Myers, S.L. Mitchell and G. Muchatibaya, Unsteady contact melting of a rectangular cross-section phase change material on a flat plate, Phys. Fluids 20 (2008) 103101, DOI:10.1063/1.2990751.

[21] T.G. Myers, Optimizing the exponent in the Heat Balance and Refined Integral Methods, Int. Commun. Heat Mass Trans. 36(2) (2009) 143-147, DOI:10.1016/j.icheatmasstransfer. 2008.10.013.

[22] T.G. Myers, Optimal exponent heat balance and refined integral methods applied to Stefan problems. Int. J. Heat Mass Trans. 53 (2010) 1119-1127, DOI:101016/j.ijheatmasstransfer.2009.10.045.

[23] T.G. Myers, S.L. Mitchell, G. Muchatibaya and M.Y. Myers A cubic heat balance integral method for one-dimensional melting of a finite thickness layer. Int. J. Heat \& Mass Trans. 50(25-26) (2007) 530-5317, DOI: http://dx.doi.org/10.1016/j.ijheatmasstransfer.2007.06.014.

[24] A. Pantokratoras, A common error made in investigation of boundary layer flows, Appl. Math. Model. 33 (2009), 413-422. DOI: doi:10.1016/j.apm.2007.11.009.

[25] Z. Rotem, A note on boundary layer solutions for pseudoplastic fluids, Chem. Engng Sci. 21 (1966), 618-620. 
[26] H. Schlichting \& K. Gersten, Boundary layer theory. 8th Edition, Springer, 2000.

[27] W.R. Schowalter, The application of boundary layer theory to power law pseudoplastic fluids: similar solutions. AIChE J. 6 (1960), 24-28.

[28] T.Y. Wang Mixed convection from a vertical plate to non-newtonian fluids with uniform surface heat-flux. Int. Comm. Heat Mass Trans. 22(3) (1995), 369-380.

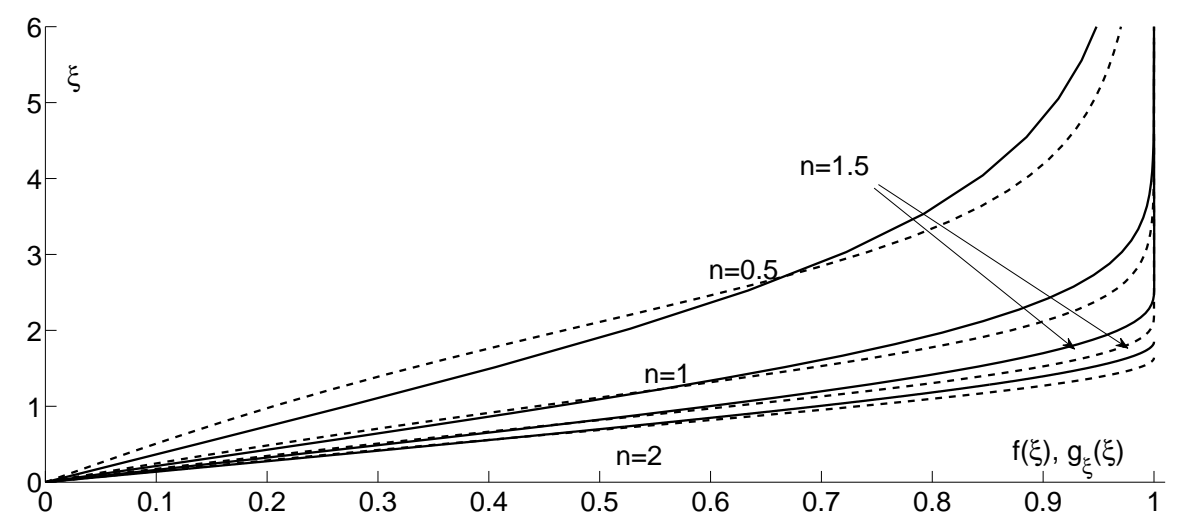

Figure 1: Comparison of similarity solutions for velocity u from equations (25), (26) 




Figure 2: Comparison of solutions for $n=1$, Blasius (solid), IME (dashed), $p=3$ (dotted), optimal $p=3.48$ (dash-dot), Pohlhausen (+)

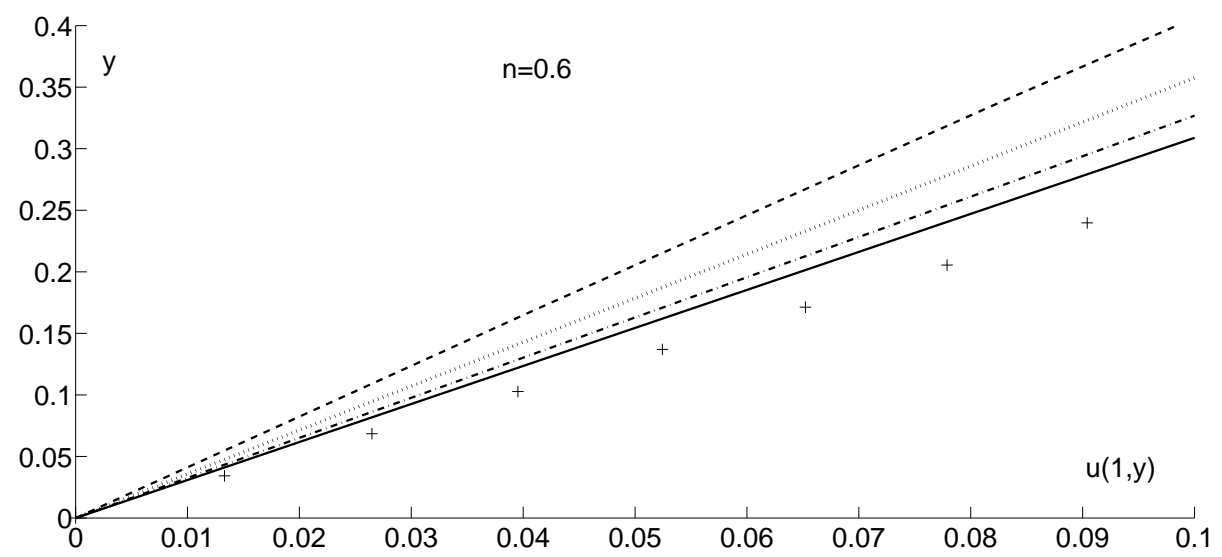

Figure 3: Comparison of solutions for $n=0.6$, refined Blasius (solid), IME (dashed), $p=3$ (dotted), optimal $p=10.02$ (dash-dot), Pohlhausen (+) 


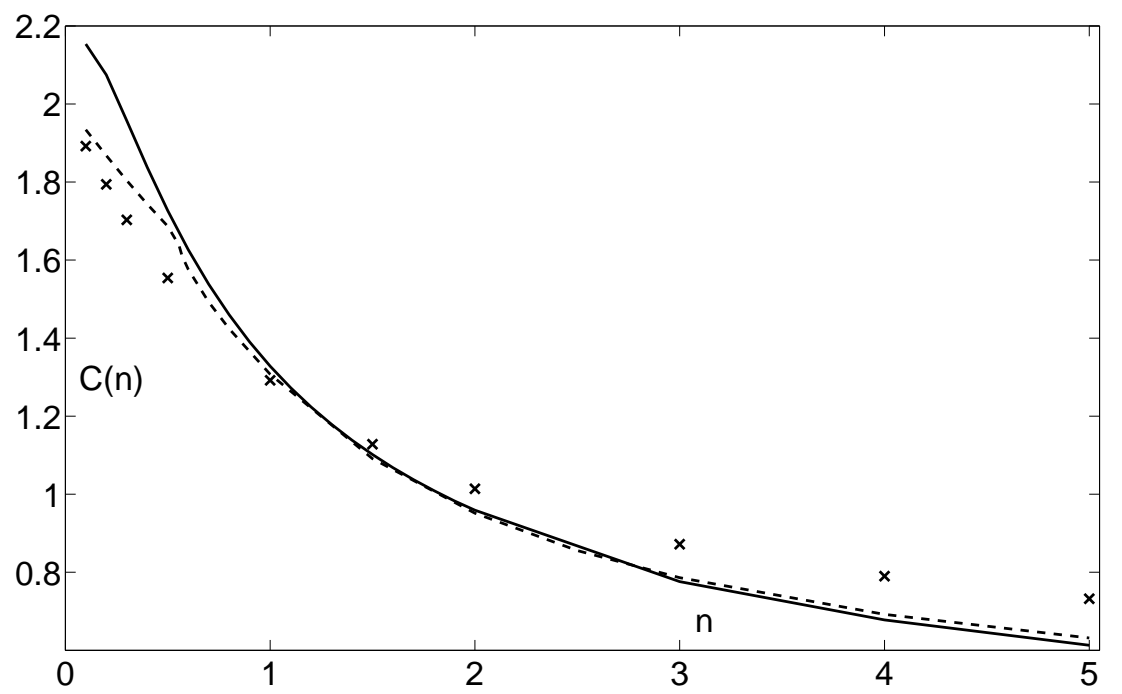

Figure 4: Drag coefficient $C(n)$ for similarity solution (solid line), optimal $p$ (dashed line) and $p=3("+")$



Figure 5: Comparison of similarity solution (solid line), IEE (dotted line) and optimal $q$ solution, $q \approx 13$ (dashed line) when $n=1$ 




Figure 6: Comparison of temperatures for $n=0.5, q=14.85, n=1, q=13, n=1.5, q=$ 12.96 (solid line) and quadratic profile (dashed line)

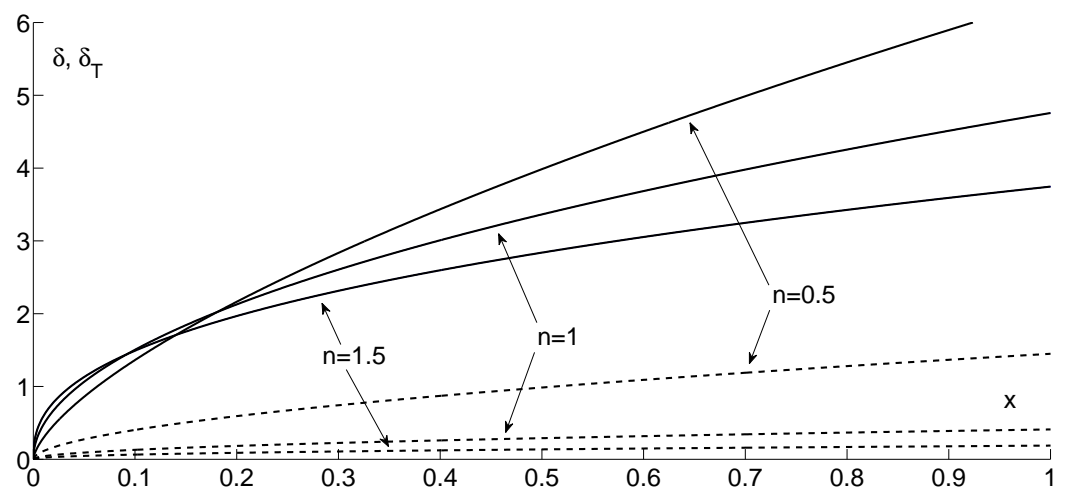

Figure 7: Development of momentum and thermal boundary layers, $\delta$ (solid line), $\delta_{T}$ (dashed line) for constant boundary temperature 


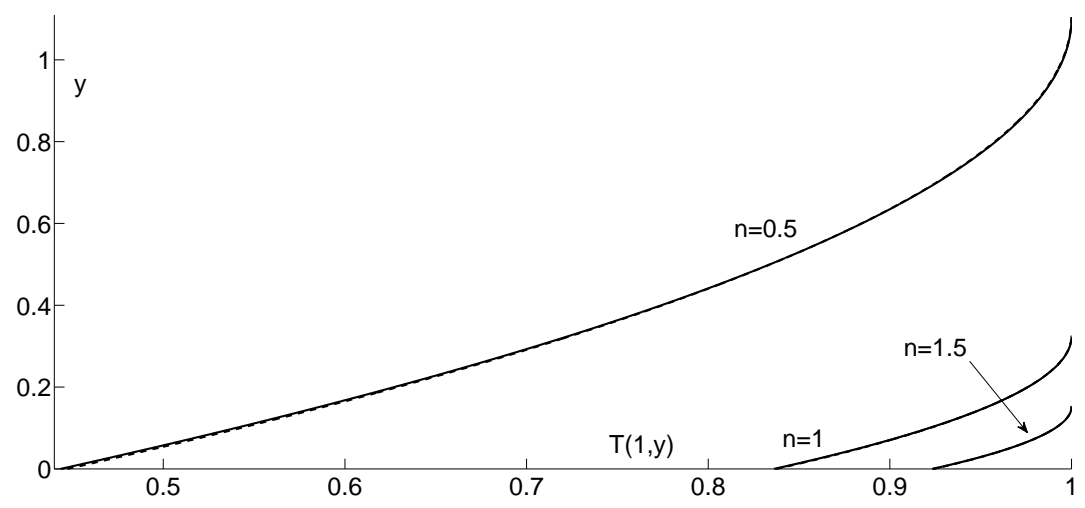

Figure 8: Comparison of temperatures with optimal $q$ (solid) and quadratic formula (dashed) for constant flux condition with $n=0.5,1,1.5$



Figure 9: Temperatures with quadratic formula (dashed) for Newton cooling condition with $n=0.5,1,1.5$ 